\title{
Social consequences of sleep disordered breathing on patients and their partners: a controlled national study
}

\author{
Poul Jennum', Rikke Ibsen² and Jakob Kjellberg ${ }^{3}$
}

\author{
Affiliations: \\ 'Danish Center for Sleep Medicine, Dept of Clinical Neurophysiology, Center for Healthy Ageing, Faculty of \\ Health Sciences, University of Copenhagen, Glostrup Hospital, Copenhagen, \\ ${ }^{2}$ itracks, Aarhus, \\ ${ }^{3}$ Danish Institute for Local and Regional Government Research, Copenhagen, Denmark.
}

Correspondence: P. Jennum, Danish Center for Sleep Medicine, Dept of Clinical Neurophysiology, Faculty of Health Sciences, University of Copenhagen, Glostrup Hospital, DK 2600 Glostrup, Denmark. E-mail: POJEAglo.regionh.dk

ABSTRACT We aimed to evaluate the total costs to patients and their partners of sleep apnoea and obesity hypoventilation syndrome (OHS) and their treatment, as this is poorly described in families.

Using data from the Danish National Patient Registry and other public databases, all patients and their partners with a diagnosis of sleep apnoea $(n=30278)$ or OHS $(n=1562)$ were included. They were compared with age-, sex- and community location-matched citizens at a ratio 1:4 (120 506 and 6241 control subjects, respectively). Direct and indirect costs were evaluated for patients and their partners.

Sleep apnoea and OHS patients and their partners had higher rates of health-related contact, medication use and unemployment, and lower income levels. Excess yearly direct net health and foregone earnings (indirect costs) were $€ 2174$ and $€ 7981$ prior to diagnosis, and $€ 3988$ and $€ 12022$ after diagnosis for sleep apnoea and OHS, respectively. The comparable annual mean excess health-related costs for spouses were $€ 1965$ and $€ 2862$ before diagnosis, and $€ 2307$ and $€ 3079$ after diagnosis, for sleep apnoea and OHS patients, respectively. These socioeconomic consequences were present up to 12 years before first diagnosis, and increased as the disease advanced.

Sleep-disordered breathing has major socioeconomic consequences for patients and their spouses years before and after diagnosis.

@ERSpublications

Sleep-disordered breathing has a major socioeconomic impact on patients and their spouses years pre- and post-diagnosis http://ow.ly/qbgaR

Received: Oct 212012 | Accepted after revision: Feb 052013 | First published online: April 052013

Support statement: The study was supported by an unrestricted grant from the Respironics Foundation and by a grant from the Centre for Healthy Ageing at the University of Copenhagen.

Conflict of interest: None declared.

Copyright @ERS 2014 


\section{Introduction}

Sleep disordered breathing (SDB), in terms of obstructive and central sleep apnoea, and obesity hypoventilation syndrome (OHS) are common treatable disorders that affect a significant proportion of the population [1]. They carry a major attributable risk of cardio- and cerebrovascular morbidity and mortality, and detrimental consequences for employment, social function and quality of life [2-7].

Despite progress in diagnosis and management, and considerable evidence of the negative effect of SDB on health, many patients with sleep apnoea and OHS are still undiagnosed or diagnosed late, and may experience secondary consequences due to later management and treatment [8]. However, the data for evaluating the consequences for employment, pension, morbidity, mortality, heath costs, costs and foregone earnings are limited. Previous studies have presented evidence of the societal burden of SDB in terms of estimates from questionnaires/quality-of-life evaluations [9-11], model-based estimates [12-14] and factual cost estimates based on national samples [2]. The occurrence and severity of SDB puts it among the most costly of disorders [15]. The burden of SDB arises not only from the disease itself and its complications, but also from the even more significant indirect social consequences, in terms of reduced ability to work [16]. Furthermore, despite the family impact of SDB [17, 18] on quality of life and sleep/daytime functioning, there are very limited data regarding the health and social consequences for the spouses of patients with sleep apnoea and OHS.

We have previously shown the significant societal burden of SDB [2], but the effect on spouses of patients with sleep apnoea or OHS has not been addressed in conjunction with economic information about SDB [19]. We aimed to evaluate the health and social consequences and consequently the health costs, foregone earnings and social transfer payments over a longer period in a larger national sample. The purpose of this paper is to focus on the factual national total health cost per case among identified patients, independent of treatment level, using a controlled design.

\section{Methods}

In Denmark, all hospital contacts are recorded in the National Patient Registry (NPR) in terms of the time of contact and including information about primary and secondary diagnoses [20]. The NPR includes administrative information, diagnoses, and diagnostic and treatment procedures using several international classification systems, including the International Classification of Diseases (ICD)-10. The NPR is a timebased national database that includes data from all inpatient and outpatient contacts, so the data that we extracted are representative of all patients in Denmark who have received a diagnosis of sleep apnoea or OHS, irrespective of other diagnoses. As data are available for the entire observation period, we can trace patients retrospectively and prospectively relative to the time of their diagnosis. Furthermore, all contacts in the primary sector (general practice and specialist care in the primary sector) and the use of medications are recorded in the national health security and the Danish Health and Medicines Authority databases, respectively. There is a small risk of underestimating the number of patients with sleep apnoea or OHS, since those with a contact in the primary sector but not in the secondary sector are recorded as having had contact but not as having received a diagnosis. Since 2009, all patients with SDB have been obliged to be recorded in the national quality database, which is based on registrations in the NPR. The economic consequences of SDB for patients and their partners were estimated by determining the yearly cost of illness per patient diagnosed with sleep apnoea (ICD-10 DG473x) and OHS (ICD-10 E662A), and comparing these figures with the cost of healthcare in a matched control group. Diagnoses of sleep apnoea and obesity hypoventilation are presented to the NPR using information from public and private hospitals. These diagnoses rely on clinical information and results of diagnostic procedures (e.g. polysomnography and partial polygraphy). The procedures are registered, but the results of the diagnostic procedures are not recorded in the NPR. The health cost was then divided into annual direct and indirect healthcare costs.

Direct costs included the average national costs of hospitalisation and outpatient cost, weighted by use, for separate diagnosis-related groups, and specific outpatient costs. These costs were all calculated from Danish Ministry of Health data using diagnosis-related groups and are average case-mix costs of hospitals or outpatient costs updated on a yearly basis. The use and costs of drugs were obtained from national Danish Health and Medicines Authority data, consisting of the retail price of each drug (including dispensing costs) multiplied by the number of transactions. The frequencies and costs of consultations with general practitioners and other specialists were based on national health security data.

Indirect costs, measured from the perspective of societal costs, included those related to a reduced labour supply and to social transfer payments. In Denmark, social transfer payments comprise income derived from state coffers. These payments include subsistence allowances, pensions, social security, social assistance, publicly funded personal support for education and others. Indirect costs were based on income figures from Coherent Social Statistics (Statistics Denmark, Copenhagen, Denmark). The partners were identified 
via Coherent Social Statistics using information about marital status or whether they shared an address. A comparable control group was identified for the spouses in a similar way to that for the patients. All information from all databases for the same period was obtained for spouses of patients and of the controls. Costs were measured on a yearly basis and adjusted to 2009 prices using Statistics Denmark's general price index for all costs. All costs were measured in Danish kroner (DKK) and converted to euros (€1:DKK7.45).

Cost-of-illness studies measure the economic burden resulting from disease and illness across a defined population, and include direct and indirect costs. Direct costs are the value of resources used in the treatment, care and rehabilitation of people with the condition under study. Indirect costs represent the value of economic resources lost because of disease-related work disability or premature mortality. As patients leave the national data registers at the time of death, the indirect costs estimate comprises only the production loss related to disease-related work disability. It is important to distinguish costs from monetary transfer payments such as disability and welfare payments. These payments represent a transfer of purchasing power to the recipients from the general taxpayers but do not represent net increases in the use of resources and, therefore, are not included in the total cost estimate.

By reviewing the NPR, we identified all patients who received a first diagnosis of sleep apnoea or OHS from 1998 to 2009. Then, using data from the Danish Civil Registration System database (which includes information about all partners, their marital status, social factors, education, employment, incomes, pensions, etc.), we randomly selected citizens of the same age and sex as the patients. Social compensation was performed by selecting control subjects who resided in the same area of the country in which the patients lived and who had the same civil status. The ratio of control subjects to patients was 4:1. This ratio was chosen to reduce variance in the control group. Data from patients and matched control subjects who could not be identified in the Coherent Social Statistics database were excluded from the sample. More than $99 \%$ of the observations in the two groups were successfully matched. Patients and matched control subjects were followed from the year of diagnosis until 2009. If a patient or control was not present in the register on January 1 each year, then the corresponding control or patient control was not included in the dataset for that year. The absence of patients from the register is typically due to their being deceased, in prison or having emigrated to another country. All patients' partners (married or not, and irrespective of sex) were identified. A similar control group of partners was identified on the basis of age, sex and sociodemographic status.

\section{Statistical analysis}

The study was approved by the Danish Data Protection Agency. Data were handled in a manner that did not reveal the identity of any patients or control subjects, so neither individual consent nor ethical approval was required.

Statistical analysis was performed using SAS version 9.1.3 (SAS Inc., Cary, NC, USA). Statistical significance of the cost estimates was assessed by nonparametric bootstrap analysis [21].

\section{Results}

We identified 30278 patients with a diagnosis of sleep apnoea and 1562 patients with a diagnosis of OHS, and compared them with 120506 and 6241 control subjects, respectively. The age and sex distribution and

TABLE 1 Age distribution of evaluated patients

Sleep apnoea

$30278(100)$

Subjects
Age years
$<20$
$20-29$
$30-39$
$40-49$
$50-59$
$60-69$
$70-79$
$\geqslant 80$

Male

Marital or cohabiting status

Patients

Controls

$2998(9.9)$
$1339(4.4)$
$3687(12.2)$
$6621(21.9)$
$8473(28.0)$
$5347(17.7)$
$1583(5.2)$
$230(0.8)$
76.6

66.2

65.4
Obesity hypoventilation

$1562(100)$

$$
\begin{gathered}
8(0.5) \\
46(2.9) \\
156(10.0) \\
311(19.9) \\
474(30.3) \\
368(23.6) \\
161(10.3) \\
38(2.4) \\
69.9
\end{gathered}
$$

63.4 65.6

Data are presented as $\mathrm{n}(\%)$ or $\%$. 
TABLE 2 Proportions of sleep apnoea and obesity hypoventilation patients and their controls receiving healthcare, and income (after diagnosis)

\begin{tabular}{lccccc} 
& \multicolumn{2}{c}{ Sleep apnoea } & & \multicolumn{2}{c}{ Obesity hypoventilation } \\
\cline { 2 - 3 } \cline { 5 - 6 } & Patients & Controls & & Patients & Controls \\
\hline Outpatient treatment & $60.3^{* * *}$ & 27.9 & & $67.1^{* * *}$ & 31.0 \\
Inpatient treatment & $29.1^{* * *}$ & 10.5 & & $49.0^{* * *}$ & 13.4 \\
Medication & $87.2^{* * *}$ & 70.3 & & $95.0^{* * *}$ & 77.4 \\
Public health insurance & $97.9^{* * *}$ & 93.2 & & $98.2^{* * *}$ & 94.4 \\
Income from employment & $60.1^{* * *}$ & 62.6 & & $41.0^{* * *}$ & 53.6 \\
Public transfer income total & $49.2^{* * *}$ & 42.0 & & $76.0^{* * *}$ & 58.8 \\
$\quad$ Pension & 11.7 & 11.3 & & $21.3^{* * *}$ & 24.7 \\
$\quad$ Other public transfers & $27.3^{* * *}$ & 23.3 & & $46.5^{* * *}$ & 28.3 \\
Sickpay (publicly funded) & $15.6^{* * *}$ & 10.8 & & $14.8^{* * *}$ & 9.3 \\
\hline
\end{tabular}

Data are presented as \%. ${ }^{* *}: p<0.001$, by Cochran-Armitage test, bootstrapped (by each expense type, irrespective of significance of share received].

marital status of the cohort are presented in table 1. As expected, most of the patients with an initial diagnosis of sleep apnoea were middle-aged (only around a 10th were children or adolescents), with a quarter being female.

Direct costs: outpatient clinic, hospital, primary care and drug costs

More patients than control subjects were treated in outpatient clinics, hospitalised or had contact with the primary care system. More patients than control subjects were taking medication, and more patients were receiving public support for their medications (table 2). This effect was most pronounced in patients with OHS, but was also noted in sleep apnoea, in terms of absolute frequencies and compared with socialcompensated controls. The same pattern was identified among spouses: outpatient and inpatient care, uses of medication and of public transfer were all higher among spouses of sleep apnoea and OHS patients, although the differences were not significant 5-7 years following diagnosis.

\section{Indirect costs: social costs, employment rate and income}

More patients than control subjects received social services. Fewer patients with sleep apnoea or OHS than control subjects received income from employment (table 3). Effects on health, employment, income from employment and public transfer income are shown in figure 1 for sleep apnoea patients and their spouses in and figure 2 for OHS patients and their spouses. Overall, the employment rate decreased with age in all groups, and there were no differences in employment rates between the groups. However, compared with control subjects, the employment rates for patients with sleep apnoea, especially for those with OHS, were

TABLE 3 Proportions of partners of patients and controls receiving healthcare, and income (after diagnosis)

\begin{tabular}{lccccc} 
& \multicolumn{2}{c}{ Sleep apnoea } & & \multicolumn{2}{c}{ Obesity hypoventilation } \\
\cline { 2 - 3 } \cline { 5 - 5 } \cline { 5 - 5 } & Partner & Control & & Partner & Control \\
\hline Outpatient treatment & $36.3^{* * *}$ & 31.0 & & $35.7^{* * *}$ & 32.9 \\
Inpatient treatment & $13.6^{* * *}$ & 11.3 & & 14.3 & 13.9 \\
Medication & $85.5^{* * *}$ & 80.6 & & $84.0^{* * *}$ & 80.2 \\
Public health insurance & $98.1^{* * *}$ & 97.1 & & 96.2 & 96.7 \\
Income from employment & $68.4^{* * *}$ & 70.4 & & 56.8 & 60.1 \\
Public transfer income total & $51.0^{* * *}$ & 45.8 & & $60.8^{* * *}$ & 54.6 \\
$\quad$ Pension & $9.6^{* * *}$ & 10.1 & & $19.0^{* * *}$ & 20.4 \\
$\quad$ Other public transfers & $30.0^{* * *}$ & 26.2 & & $33.0^{* * *}$ & 25.0 \\
$\quad$ Sickpay (publicly funded) & $17.0^{* * *}$ & 14.1 & & $13.3^{* * *}$ & 12.8 \\
\hline
\end{tabular}

Data are presented as \%. ${ }^{* * *}: \mathrm{p}<0.001$, by Cochran-Armitage test, bootstrapped (by each expense type, irrespective of significance of share received). 

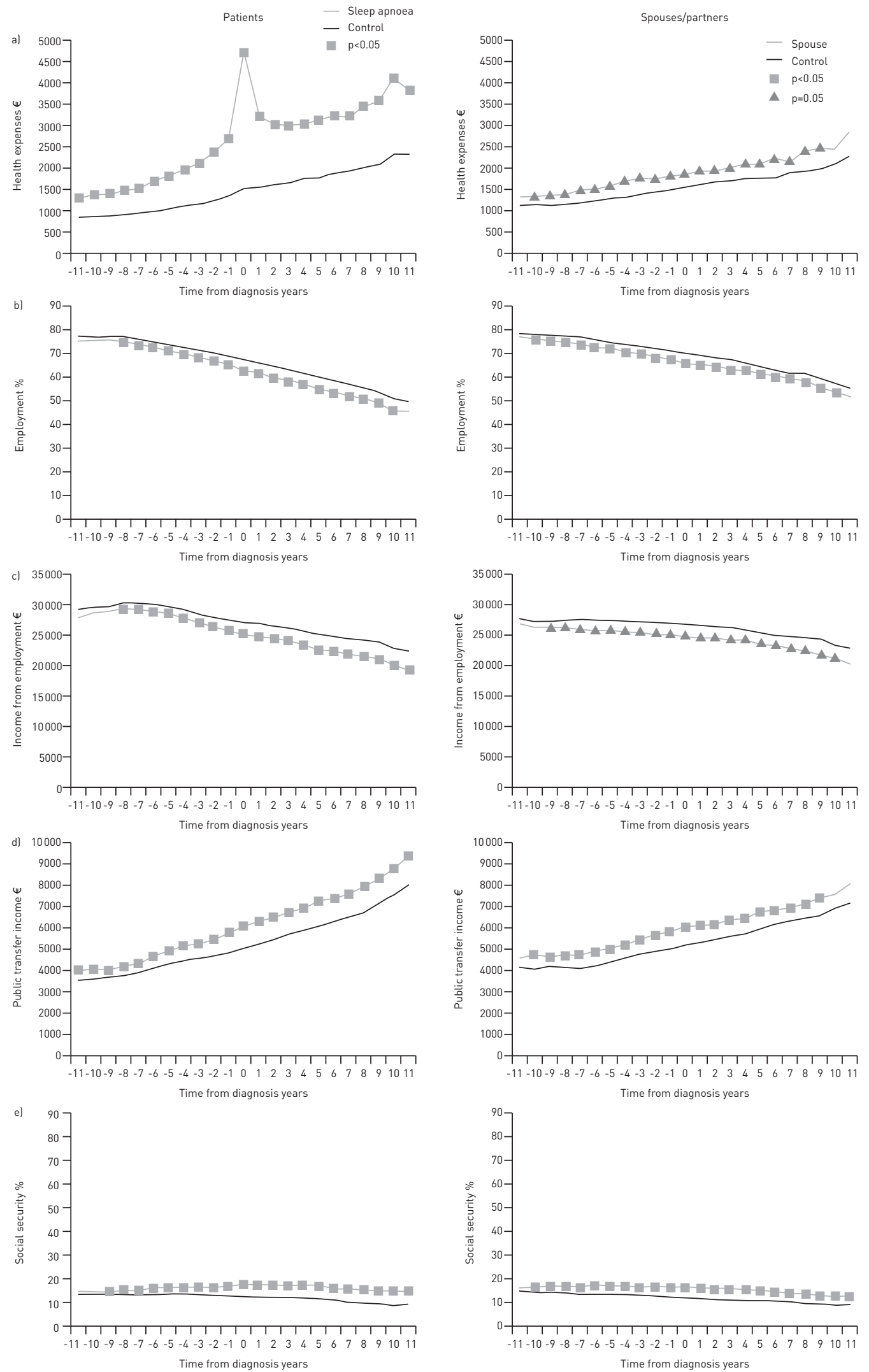

FIGURE 1 Effect of sleep-disordered breathing on a) health expenses; b) employment; c) income from employment; social income; d) public transfer income; and e) rate of social security in patients with sleep apnoea and their spouses/ partners. 
Patients

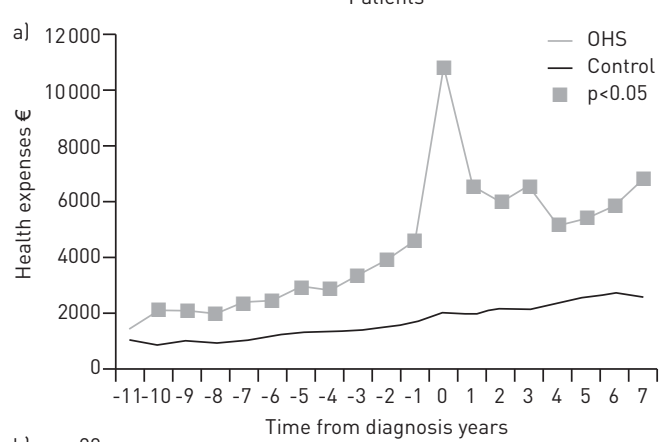

b)
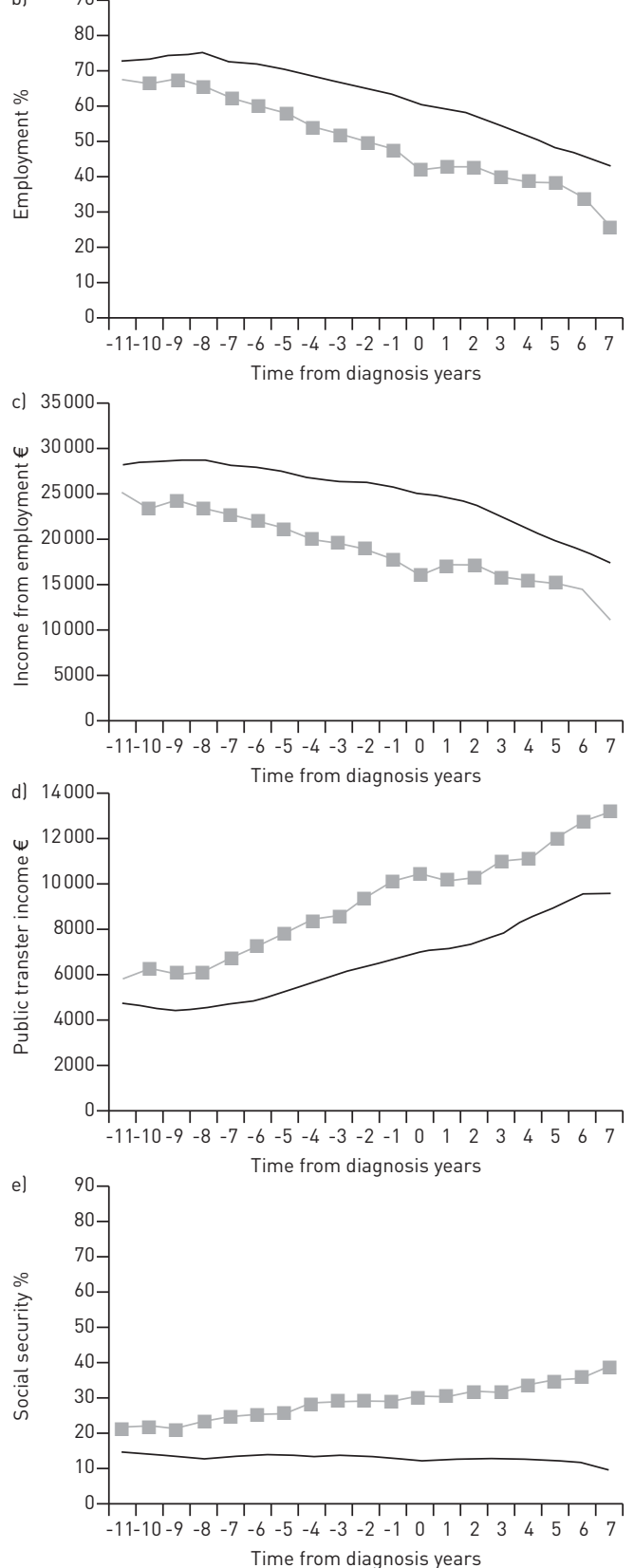

Spouses/partners
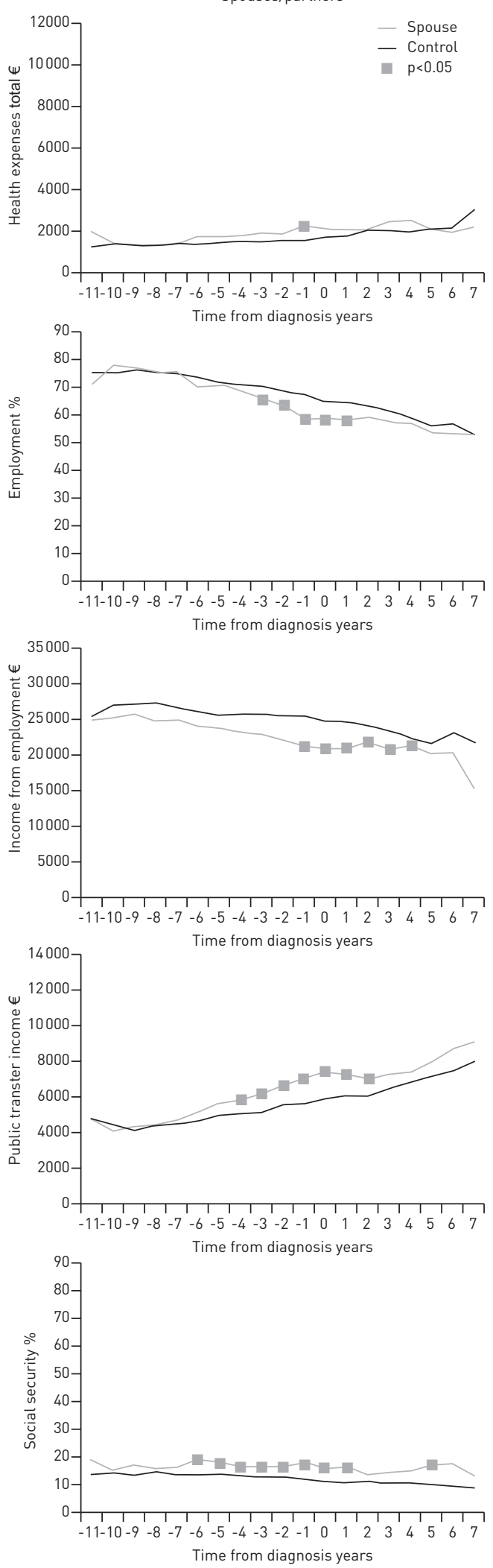

FIGURE 2 Effect of sleep disordered breathing on a) health expenses; b) employment; c) income level; social income; d) public transfer income; and e) rate of social security in patients with obesity hypoventilation syndrome (OHS) and their spouses/partners. Only 7 years of follow-up are shown due to limits in the number of cases. 
significantly lower up to 12 years before diagnosis and decreased further after a diagnosis had been made. In addition, a corresponding increase in social transfer expenses was made to patients. The rate of retirement was similar in subjects with and without sleep apnoea, but in OHS patients, the rate of patients going on to receive a pension decreased slightly, probably due to higher mortalities (not shown). The corresponding expenses in total direct and indirect costs were elevated before a diagnosis of sleep apnoea and OHS were made, and increased further afterwards. The social implications of OHS are particularly well illustrated by the much lower employment rate and higher total direct and indirect costs in the OHS control group (selection made by social compensation). The peak in expenses in sleep apnoea and OHS patients at the time of all diagnoses is due to the direct costs related to disease management (diagnosis and treatment).

\section{Total health costs per year}

The sources of information and the average annual health cost per person year by cost categories in Denmark, compared with that of age-, sex- and socioeconomic status-matched control subjects, are presented in table 4. Excess direct net health costs (general practitioner services, hospital services and medication) and indirect costs (loss of labour market income), including social transfer payments for patients compared with control subjects were $€ 2821$ for sleep apnoea and $€ 10463$ for OHS before diagnosis and $€ 5060$ and $€ 15001$, respectively, after diagnosis. The comparable annual mean excess healthrelated costs for spouses (table 5) were $€ 2639$ (sleep apnoea) and $€ 3523$ (OHS) before diagnosis, and $€ 3058$ and $€ 4068$, respectively, after diagnosis.

\section{TABLE 4 Share of patients and controls receiving healthcare, and income}

Sleep apnoea

\section{Patients}

188074
$264^{* * *}$
$939^{* * *}$
$454^{* * *}$
$322^{* * *}$
$27727 * * *$
$4988^{* * *}$
$906 * * *$
$3332^{* * *}$
$750^{* * *}$
$3275^{* * *}$
$1980^{* * *}$
1295
2174
$4988^{* * *}$

2821

Net costs including transfers

\section{After diagnosis}

Observations

Outpatient treatment

In patient treatment

Medication

Public health insurance

Income from employment

Public transfer income total

Pension

Other public transfers

Sickpay (publicly funded)

Total costs

Direct health costs

Indirect costs, foregone earnings

Net costs

Social transfer payments

Net costs including transfers
157116

$585^{* * *}$

$1798^{* * *}$

$666^{* * *}$

$413^{* * *}$

$23798^{* * *}$

$6778 * * *$

$2228 * * *$

$3789 * * *$

$761^{* * *}$

$5683 * * *$

$3462^{* * *}$

2221

3988

$6778^{* * *}$

5060
Obesity hypoventilation

Controls

Patients

Controls

Data are presented as $\mathrm{n} .{ }^{* * *}: \mathrm{p}<0.001$, calculated by $\mathrm{t}$-test and bootstrapped.

744371
162
504
237
199
29023
4341
861
3007
473
1101
1101


4341

628766
285
839
326
245
26018
5706
2122
3113
471
1695
1695

5706

11797

$332^{* * *}$

$1565^{* * *}$

$797 * * *$

$344^{* * *}$

20981

$7971^{* * *}$

$1677^{* * *}$

$5368^{* * *}$

$926 * * *$

$9291 * * *$

$3037^{* * *}$

$6254^{* * *}$

$7981^{* * *}$

$7971^{* * *}$

10463

5644

$951 * * *$

4863***

$1251^{* * *}$

$483^{* * *}$

$16073^{* * *}$

$10794^{* * *}$

3023***

$6774^{* * *}$

$997^{* * *}$

$14239 * * *$

6692

12022

$10794^{* * *}$

15001
46859

186

627

295

203

27236

5488

1570

3360

558

1311

1311

5489

26127

345

1159

429

284

22765

7816

3734

3552

530

2216

2216

7816 
TABLE 5 Frequency of partners of patients with sleep apnoea and obesity hypoventilation and their controls receiving healthcare, and income

\begin{tabular}{|c|c|c|c|c|}
\hline & \multicolumn{2}{|c|}{ Sleep apnoea } & \multicolumn{2}{|c|}{ Obesity hypoventilation } \\
\hline & Partners & Controls & Partners & Controls \\
\hline Observations & 126922 & 502423 & 7564 & 32046 \\
\hline Outpatient treatment & $257 * * *$ & 206 & $262^{* * *}$ & 217 \\
\hline Inpatient treatment & $732^{* * *}$ & 602 & $831^{\#}$ & 702 \\
\hline Income from employment & 25680 & 27342 & $23492^{* * *}$ & 26030 \\
\hline Public transfer income total & $5217^{* * *}$ & 4544 & $5644^{* * *}$ & 4947 \\
\hline Pension & 681 & 702 & $934^{* * *}$ & 1102 \\
\hline Other public transfers & $3525^{* * *}$ & 2991 & $3779 * * *$ & 3042 \\
\hline Sickpay (publicly funded) & $1011^{* * *}$ & 851 & $931 * * *$ & 803 \\
\hline Total costs & $3285^{* * *}$ & 1319 & $4280 * * *$ & 1454 \\
\hline \multicolumn{5}{|l|}{ After diagnosis } \\
\hline Number of observations & 103833 & 414510 & 3525 & 16642 \\
\hline Outpatient treatment & $396^{* * *}$ & 331 & 391 & 389 \\
\hline Inpatient treatment & $861^{* * *}$ & 746 & 979 & 930 \\
\hline Medication & $418^{* * *}$ & 336 & $482^{* * *}$ & 378 \\
\hline Public health insurance & $359 * * *$ & 296 & 322 & 293 \\
\hline Income from employment & $24084^{* * *}$ & 26067 & 20812 & 23707 \\
\hline Public transfer income total & $6412^{* * *}$ & 5661 & 7431 & 6442 \\
\hline Pension & $1637^{\#}$ & 1695 & 2348 & 2554 \\
\hline Other public transfers & $3778^{* * *}$ & 3151 & $4208^{* * *}$ & 3136 \\
\hline Sickpay (publicly funded) & $997 * * *$ & 816 & 875 & 752 \\
\hline Total costs & $4016^{* * *}$ & 1709 & $5069 * * *$ & 1990 \\
\hline Direct health costs & $2034^{* * *}$ & 1709 & 2174 & 1990 \\
\hline
\end{tabular}

Data are presented as $n .{ }^{* *}: p<0.001,{ }^{*}: p=0.02$, calculated by t-test and bootstrapped.

Influence of age and sex on employment and direct and indirect costs

For all ages, the direct costs were higher in patients, especially those with sleep apnoea and OHS. Both sleep apnoea and OHS patients, especially females (data not shown), had lower incomes at all ages.

\section{Discussion}

This study shows that sleep apnoea and especially OHS have a significant socioeconomic impact on patients and their partners, compared with a random population-based sample controlled for socioeconomic status. These effects are present several years before a diagnosis is established. The current study uses factual cost estimates in contrast to previous estimates, which applied measures of selected patient groups without controls and involved financial modelling or quality estimates.

The results confirm our previous finding that patients with sleep apnoea and OHS had two to three times greater medication and hospital costs, had more than twice the total health costs, and had employment rates that were $>30 \%$ lower. Patients with OHS had the lowest employment rate. Employed patients tended to earn only two-thirds of the income of control subjects, with OHS patients having the lowest earnings. Patients with sleep apnoea, and especially OHS, had much higher social expenditures than control subjects. Thus, sleep apnoea, and especially OHS, has a major impact on social outcomes, including employment. This is probably due to a complex interaction between pre-existing social factors, adiposity, comorbidity and SDB, which has a major influence on socioeconomic status and employment level. The study further documents the effect on patients' spouses; those of both OHS patients and sleep apnoea patients had the 
highest healthcare usage and encountered the greatest social effect. Consequently, SDB has a major impact not only on the quality of life, but also on the health status and social outcome of patients' partners.

Previous studies have shown that family members may be affected by the patient's sleep apnoea in terms of reduced quality of life, sleep complaints, daytime functioning and health consequences [17, 22-28]. However, the health burden and that of partners, compared with controls, has not been systematically evaluated before. Determining the economic consequences of SDB is complex. The current study adjusts for demographic variables, which is why the results presented here are probably quite conservative when compared with those from the general population. Sleep apnoea, and especially OHS, and adherence to continuous positive airway pressure (CPAP) treatment are associated with social factors [2, 4, 7, 29-32]. Financial enticements influence patients' decisions to accept or purchase CPAP devices [33] and minimising cost sharing reduces a barrier for CPAP acceptance among patients with low socioeconomic status [34]. In Denmark, diagnosis and treatment are paid for by public insurance, but despite this, obstructive sleep apnoea and OHS are probably underdiagnosed, especially among lower socioeconomic groups. With an accurate diagnosis and appropriate treatment, patients reduce their risk of complications in terms of cardiovascular disease and the associated costs, but diagnostic procedures, treatment and management of SDB add to the direct costs. However, even when we included the costs associated with the diagnosis and treatment of SDB, we found that patients with SDB incur a significant socioeconomic burden because their lower employment rates and the lower earning potential of those who are employed exceed the direct costs of the disease for patients and spouses.

Patients and their partners had already experienced a significantly reduced employment status and higher level of direct and indirect costs before diagnosis, and there were further falls thereafter. This finding is most pronounced in patients with OHS, and is probably due to pre-existing disease manifestations, comorbidities, etc. [2,35]. The finding that the social uses increases especially in OHS patients after diagnosis implies that these patients have not been able to seek a pension before they received their diagnosis, and/or that the patients have reached a level of exhaustion. Health consequences and retirement and unemployment have the strongest effect among the youngest people, in whom the impact of indirect costs such as work ability is most pronounced. This is an important factor for disease identification and for planning health care. For example, as disease management (prevention and treatment) of SDB is expected to affect direct and indirect costs, early intervention would be most important in chronic diseases, such as those in which there is the possibility of influencing secondary factors (e.g. cardiovascular, social or cognitive morbidities) because the patient's functionality is already affected by the time of diagnosis. This may explain, in part, why the current treatment modalities of SDB have only limited economic value in the short term as the influence on direct and indirect cost is weaker when an accurate diagnosis of SDB is delayed, clearly highlighting the need for early detection and intervention. In this study, we have not evaluated the economic effect of CPAP treatment, but in a previous study we showed a positive effect of CPAP treatment on mortality in sleep apnoea patients but not on health economics over a shorter observation period (2 years before and after diagnosis) [2]. This is to be expected, since the effect of treatment should counterbalance the cost of the diagnostic and initial treatment procedures as well as the indirect costs. However, health economy depends not only on the reduction in mortality, but also on morbidity and the ability to remain in employment. As employment and health are affected before a current diagnosis, this argues for early detection and disease management. SDB is so common, affecting a wide range of males and females, that general health recommendations are advised, in addition to health campaigns [36].

Previous studies have suggested that partners of patients with sleep apnoea also suffer in terms of daytime symptoms and worry [18], and reduced quality of life [17], although this may improve after CPAP treatment $[24,25]$. Our study further suggests that SDB also has health and social implications beyond what can be explained solely by social selection.

There are some limitations to this study: 1) the accuracy of diagnosis and management are sensitive to the diagnostic criteria used by the reporting doctors; 2) the local management of treatment, especially CPAP, may be under-reported; 3) obstructive sleep apnoea and OHS are underestimated in the control group, so the data presented are a conservative estimate; and 4) although we have included the cost of CPAP, we have not reported detailed information about usage and effect. Due to the burden of disease, the clinical variation in disease management and often low compliance and follow-up [32, 37], it is worth considering setting up a national quality database, as has been done in Denmark. This was established in 2009 and clinics report apnoea severity and effect of treatment. These data are not complete for the entire period, and are therefore not reported. Conversely, the strengths of the study are that all patients and their spouses can be identified, and the actual health, income level and social usage can be identified due to national time-locked registrations of all citizens. 
In conclusion, the current study, which includes data from all national patients diagnosed with sleep apnoea and OHS over a 12-year period in Denmark, found that these disorders lead to significantly higher health-related and social transfer costs, and lower levels of employment and income among patients and their partners. The study also identified a significant health-related impact and socioeconomic aspect of these conditions, suggesting a need to address further the relevance of early diagnosis of SDB and measuring the effect of treatment. The study argues for earlier disease identification and management to reduce these consequences.

\section{References}

1 Jennum P, Riha RL. Epidemiology of sleep apnoea/hypopnoea syndrome and sleep-disordered breathing. Eur Respir J 2009; 33: 907-914.

2 Jennum P, Kjellberg J. Health, social and economical consequences of sleep-disordered breathing: a controlled national study. Thorax 2011; 66: 560-566.

3 Laaban JP, Mounier L, Roque d'Orbcastel O, et al. Cardiovascular risk factors in men and women with obstructive sleep apnoea syndrome. Respir Med 2010; 104: 1063-1068.

4 Li X, Sundquist K, Sundquist J. Socioeconomic status and occupation as risk factors for obstructive sleep apnea in Sweden: a population-based study. Sleep Med 2008; 9: 129-136.

5 Martínez-García MA, Soler-Cataluña JJ, Ejarque-Martínez L, et al. Continuous positive airway pressure treatment reduces mortality in patients with ischemic stroke and obstructive sleep apnea: a 5-year follow-up study. Am J Respir Crit Care Med 2009; 180: 36-41.

6 Punjabi NM, Caffo BS, Goodwin JL, et al. Sleep-disordered breathing and mortality: a prospective cohort study. PLoS Med 2009; 6: e1000132.

7 Tarasiuk A, Greenberg-Dotan S, Simon T, et al. Low socioeconomic status is a risk factor for cardiovascular disease among adult obstructive sleep apnea syndrome patients requiring treatment. Chest 2006; 130: 766-773.

8 Kapur V, Blough DK, Sandblom RE, et al. The medical cost of undiagnosed sleep apnea. Sleep 1999; 22: 749-755.

9 Gander P, Scott G, Mihaere K, et al. Societal costs of obstructive sleep apnoea syndrome. NZ Med J 2010; 123 : 13-23.

10 Pagel JF. Obstructive sleep apnea (OSA) in primary care: evidence-based practice. J Am Board Fam Med 2007; 20: 392-398.

11 Pietzsch JB, Garner A, Cipriano LE, et al. An integrated health-economic analysis of diagnostic and therapeutic strategies in the treatment of moderate-to-severe obstructive sleep apnea. Sleep 2011; 34: 695-709.

12 Guest JF, Helter MT, Morga A, et al. Cost-effectiveness of using continuous positive airway pressure in the treatment of severe obstructive sleep apnoea/hypopnoea syndrome in the UK. Thorax 2008; 63: 860-865.

13 Tan MC, Ayas NT, Mulgrew A, et al. Cost-effectiveness of continuous positive airway pressure therapy in patients with obstructive sleep apnea-hypopnea in British Columbia. Can Respir J 2008; 15: 159-165.

14 Tripathi A, Jerrell JM, Stallworth JR. Cost-effectiveness of adenotonsillectomy in reducing obstructive sleep apnea, cerebrovascular ischemia, vaso-occlusive pain, and ACS episodes in pediatric sickle cell disease. Ann Hematol 2011; 90: 145-150.

15 Gustavsson A, Svensson M, Jacobi F, et al. Cost of disorders of the brain in Europe 2010. Eur Neuropsychopharmacol 2011; 21: 718-779.

16 Fietze I. The Danish study on cost effectiveness in sleep related breathing disorders - a possible example for Europe. Thorax 2011; 66: 556-558.

17 Breugelmans JG, Ford DE, Smith PL, et al. Differences in patient and bed partner-assessed quality of life in sleepdisordered breathing. Am J Respir Crit Care Med 2004; 170: 547-552.

18 Virkkula P, Bachour A, Hytönen M, et al. Patient- and bed partner-reported symptoms, smoking, and nasal resistance in sleep-disordered breathing. Chest 2005; 128: 2176-2182.

19 Simon-Tuval T, Reuveni H, Greenberg-Dotan S, et al. Low socioeconomic status is a risk factor for CPAP acceptance among adult OSAS patients requiring treatment. Sleep 2009; 32: 545-552.

20 Lynge E, Sandegaard JL, Rebolj M. The Danish National Patient Register. Scand J Public Health 2011; 39: Suppl. 7 , $30-33$.

21 Efron B, Tibshirani RJ. An Introduction to the Bootstrap. London, Chapman \& Hall, 1993.

22 Ashtyani H, Hutter DA. Collateral damage: the effects of obstructive sleep apnea on bed partners. Chest 2003; 124: 780-781.

23 Beninati W, Harris CD, Herold DL, et al. The effect of snoring and obstructive sleep apnea on the sleep quality of bed partners. Mayo Clin Proc 1999; 74: 955-958.

24 Doherty LS, Kiely JL, Lawless G, et al. Impact of nasal continuous positive airway pressure therapy on the quality of life of bed partners of patients with obstructive sleep apnea syndrome. Chest 2003; 124: 2209-2214.

25 Parish JM, Lyng PJ. Quality of life in bed partners of patients with obstructive sleep apnea or hypopnea after treatment with continuous positive airway pressure. Chest 2003; 124: 942-947.

26 Smith AK, Togeiro SM, Tufik S, et al. Disturbed sleep and musculoskeletal pain in the bed partner of patients with obstructive sleep apnea. Sleep Med 2009; 10: 904-912.

27 Thomopoulos C, Michalopoulou H, Kasiakogias A, et al. Resistant hypertension and obstructive sleep apnea: the sparring partners. Int J Hypertens 2011; 2011: 947246.

28 Uloza V, Balsevicius T, Sakalauskas R, et al. Changes in emotional state of bed partners of snoring and obstructive sleep apnea patients following radiofrequency tissue ablation: a pilot study. Sleep Breath 2010; 14: 125-130.

29 Aloia MS. Social factors associated with CPAP acceptance. Sleep 2009; 32: 443-444.

30 Charokopos N, Leotsinidis M, Tsiamita M, et al. Sleep apnea syndrome in a referral population in Greece: influence of social factors. Lung 2007; 185: 235-240.

31 McDowell A. Spousal involvement and CPAP adherence: a two-way street? Sleep Breath 2011; 15: 269-270.

32 Paine SJ, Harris RB, Mihaere KM. Managing obstructive sleep apnoea and achieving equity: implications for health services. N Z Med J 2011; 124: 97-104.

33 Brin YS, Reuveni H, Greenberg S, et al. Determinants affecting initiation of continuous positive airway pressure treatment. Isr Med Assoc J 2005; 7: 13-18. 
34 Tarasiuk A, Reznor G, Greenberg-Dotan S, et al. Financial incentive increases CPAP acceptance in patients from low socioeconomic background. PLoS One 2012; 7: e33178.

35 Jennum P. Quality of life, co-morbidity and obstructive sleep apnoea. Clin Respir J 2010; 4: 129-130.

36 Laitinen LA, Anttalainen U, Pietinalho A, et al. Sleep apnoea: Finnish National guidelines for prevention and treatment 2002-2012. Respir Med 2003; 97: 337-365.

37 Tzischinsky O, Shahrabani S, Peled R. Factors affecting the decision to be treated with continuous positive airway pressure for obstructive sleep apnea syndrome. Isr Med Assoc J 2011; 13: 413-419. 